\title{
Role of motor function and lung function in pathways to ageing and decline
}

\author{
Deborah Finkel $^{1,2}$ (D) Marie Ernsth Bravell ${ }^{2} \cdot$ Nancy L. Pedersen ${ }^{3,4}$
}

Received: 8 November 2019 / Accepted: 22 January 2020 / Published online: 13 February 2020

(c) The Author(s) 2020

\begin{abstract}
Background Extensive research has investigated the association between age changes in various domains, including lung function and motor function. However, a few analyses have tested models that incorporate bidirectional longitudinal influences between lung and motor function to test the temporal chain of events in the disability process. Dual change score models (DCSM) assist with identification of leading indicators of change by leveraging longitudinal data to examine the extent to which changes in one variable influence subsequent changes in a second variable, and vice versa.

Aims The purpose of the current-analysis study was to apply DCSM to data from the Swedish Adoption/Twin Study of ageing to examine the nature of the longitudinal relationship between motor functioning and lung function.

Methods Three motor functioning factors were created from 20 performance measures, including measures of balance, flexibility, and fine motor skills. Peak expiratory flow measured lung function. Participants were 829 adults aged 50-88 at the first of 9 waves of testing covering a 27-year follow-up period; $80 \%$ participated in at least three waves.

Results Model comparisons indicated that decline in lung function preceded and contributed to subsequent decline in motor function.

Discussion Combined with previous results, these results suggest that declining lung function results in increasing difficulties in motor function, which contribute to subsequent declines in multiple domains.

Conclusion Understanding the cascade of events that can lead to dependence can help in the development of interventions targeted early in the disablement process.
\end{abstract}

Keywords Lung function $\cdot$ Motor function $\cdot$ Physical function $\cdot$ Longitudinal $\cdot$ Ageing $\cdot$ Disablement

Electronic supplementary material The online version of this article (https://doi.org/10.1007/s40520-020-01494-3) contains supplementary material, which is available to authorized users.

Deborah Finkel

dfinkel@ius.edu

1 Department of Psychology, Indiana University Southeast, New Albany, IN, USA

2 School of Health and Welfare, Jönköping University, Jönköping, Sweden

3 Department of Medical Epidemiology and Biostatistics, Karolinska Institute, Stockholm, Sweden

4 Department of Psychology, University of Southern California, Los Angeles, CA, USA

\section{Introduction}

Documented increases in lifespan can translate to an intensified challenge to minimize years lived with morbidity and dependence, and to maximize quality of the additional years of life [1, 2]. Physical functioning and cognitive functioning are essential to the maintenance of independence in older individuals, particularly the ability to remain mobile [3]. Mobility measures such as balance and gait have been shown to predict the onset of difficulties with activities of daily living that are foundational to independent living [4, 5]. Identification of the causal chain that leads from independence to dependence may enable health practitioners to identify older adults at risk for dependence and to implement appropriate interventions at the optimal point in the progression.

Models of the disablement process posit multiple factors that lead to eventual disability, highlighting a pathway from impairments of function (musculoskeletal and 
cardiovascular) to functional limitations (walking and balancing) to disability [6]. Extensive research has focused on the relationship between physical function and functional limitations, including investigations of the association between lung function and motor function. Impaired lung function can reduce the energy supply available for mobility, resulting in greater fatigue and increased time required to perform daily tasks, even if the tasks can be completed without assistance [7, 8]. Research indicates that reductions in balance and mobility are found in patients with challenges to lung function, such as chronic obstructive pulmonary disease [9]. Even in healthy older adults, lung function and respiratory muscle strength were significantly correlated with walking speed and other measures of physical performance in cross-sectional studies [3, 10, 11].

Stronger tests of the temporal chain of events in the disablement process require longitudinal data. In an early study of Swedish adults aged 84-90 years at intake, measured lung function (peak expiratory flow rate) predicted stability in self-reported mobility over 2 years, but not over 4 years of follow-up [12]. In a sample of Danish adults interviewed at ages 75 and 80 , better lung function and walking speed both contributed to prediction of self-reported mobility activities for men, but not for women [13]. Two more recent studies relied on annual assessment data from the Rush Memory and ageing project $[7,14]$. Lung function as measured by a combination of forced expiratory volume, forced vital capacity, and peak expiratory flow contributed significantly to the prediction of incident mobility (defined by measured gait speed) over an average of 4 years of follow-up, even in proportional hazard models that also included measures of respiratory and leg muscle strength [7]. In a linear mixedeffects analysis of change in walking ability over an average of 3 years, greater respiratory muscle strength was associated with slower rates of decline in mobility [14].

Taken together, these studies suggest a chain of events from lung function to mobility in late adulthood, although the average longitudinal follow-up period was at most 5 years. Moreover, none of these studies tested alternative models of the causal chain in which changes in motor function contributed to subsequent lung function. The disablement process model includes the possibility of feedback loops from disability to functional limitations and impairments [6], and studies suggest that physical function predicts age-related decline in lung function (e.g., [15]. To our knowledge, however, only one study to date has tested alternate models of the chain of events. Sillanpää and colleagues [16] used cross-sectional data to test three different path models of the relationships among muscle strength, measured mobility, and lung function. Model comparisons indicated that the model including pathways from muscle strength to lung function to mobility provided the best fit to the data.
The goal of the current analysis was to conduct a similar comparison of alternative temporal models of the relationship between lung function and motor function using longitudinal data from the Swedish Adoption/Twin Study of ageing [17]. SATSA includes up to nine waves of testing for a follow-up period of up to 27 years. In addition to standard spirometry measures, SATSA collected 24 different measures of motor function tapping balance, flexibility, and fine motor skills. These data were used to test models incorporating bidirectional longitudinal influences between lung and motor function to test the temporal chain of events in the disablement process.

\section{Methods}

\section{Participants}

Accrual procedures for the Swedish Adoption/Twin Study of ageing (SATSA) have been described previously. In brief, the sample was recruited from the population-based Swedish Twin Registry [17]. In-person testing (IPT) took place in a location convenient to the participants and was completed during a single 4-h visit. At IPT2 through IPT5, additional twins who had reached age 50 since the last wave were invited to participate in SATSA. Intervals between testing waves ranged from 2 to 7 years; the total time span from IPT1 to IPT10 was 27 years (note that IPT4 had a reduced sample and, thus, is not included in the current analyses). A total of 829 participants had both lung and motor functioning data from at least one wave; mean number of waves was $4.87(\mathrm{SD}=2.63)$ and mean follow-up was 13.54 years $(\mathrm{SD}=8.71)$. Eighty percent of the sample participated in three or more waves. Sixty percent of the sample was female and there were no sex differences in mean waves of participation $[t(827)=0.39, n . s$. $]$ or mean follow-up $[t(827)=1.12$, n.s.]. Mean ages at each wave are presented in Table 1.

Data were divided into fourteen 3-year age intervals from ages 50 to 89 . The data were divided into age intervals, such that everyone with data (regardless of wave) at ages 50-52.9 was included in the first age interval, labeled "50." Sample sizes within the age intervals are maximized in the middle of the age range (60-80). The data become too sparse after age interval "89" (i.e., after age 91.9) to support statistical modeling; therefore, only data up to age interval " 89 " were included in these analyses. Sample sizes and means for lung and motor functioning (translated to $\mathrm{T}$ scores) in each age interval are provided in Supplemental Table 1.

\section{Measures}

\section{Motor functioning}

Twenty-four measures of motor functioning were collected at each wave. Data reduction included two components 
Table 1 Sample characteristics at each wave of testing

\begin{tabular}{lllllll}
\hline Wave & $N$ & $\begin{array}{l}\text { Age } \\
\text { Mean }(S D)\end{array}$ & $\begin{array}{l}\text { PEF } \\
\text { Mean }(S D)\end{array}$ & $\begin{array}{l}\text { Balance } \\
\text { Mean }(S D)\end{array}$ & $\begin{array}{l}\text { Flexibility } \\
\text { Mean (SD) }\end{array}$ & $\begin{array}{l}\text { Fine motor } \\
\text { Mean (SD) }\end{array}$ \\
\hline IPT1 & 531 & $64.82(8.30)$ & $368.19(106.63)$ & $10.83(2.17)$ & $2.02(0.21)$ & $8.65(1.48)$ \\
IPT2 & 551 & $65.74(8.91)$ & $370.49(108.95)$ & $10.70(1.97)$ & $2.19(0.52)$ & $8.55(1.21)$ \\
IPT3 & 546 & $68.64(9.20)$ & $366.32(126.83)$ & $10.98(2.14)$ & $2.25(0.58)$ & $8.67(1.31)$ \\
IPT5 $^{\mathrm{a}}$ & 506 & $70.11(9.66)$ & $339.97(139.23)$ & $11.74(3.75)$ & $2.29(0.77)$ & $9.02(2.50)$ \\
IPT6 & 398 & $71.35(8.77)$ & $363.75(146.87)$ & $11.76(3.30)$ & $2.32(0.71)$ & $9.31(2.23)$ \\
IPT7 & 348 & $73.95(8.74)$ & $359.09(131.15)$ & $12.34(4.21)$ & $2.45(0.87)$ & $9.81(3.39)$ \\
IPT8 & 301 & $75.02(8.12)$ & $366.14(130.67)$ & $13.56(5.53)$ & $2.60(1.16)$ & $-{ }^{\mathrm{b}}$ \\
IPT9 & 259 & $76.40(7.94)$ & $363.92(123.22)$ & $14.00(5.91)$ & $2.69(1.27)$ & $-{ }^{\mathrm{b}}$ \\
IPT10 & 242 & $77.70(7.56)$ & $359.68(123.17)$ & $13.59(5.20)$ & $2.48(0.87)$ & $-{ }^{\mathrm{b}}$ \\
\hline
\end{tabular}

${ }^{\mathrm{a}}$ IPT4 had insufficient data to be included in analyses

${ }^{\mathrm{b}}$ Fine motor factor was not collected at waves $8-10$
[18]. First, analyses indicated that nurse ratings of successful performance $(1=$ no difficulty, $2=$ some difficulty, and $3=$ impossible) were more sensitive to subtle changes with age than performance time $[18,19]$. In other words, timed performance of young-old adults on these measures did not vary extensively; in contrast, qualitative ratings demonstrated more variance across the entire age range included in these analyses. Therefore, nurse ratings of performance were used, instead of timing data, to generate the motor factors. Second, factor analyses were compared across wave and age to identify the most consistent and interpretable factor solution, resulting in a three-factor solution incorporating 20 of the items. The remaining four items did not load consistently on any factor. The flexibility factor included two items: touch left earlobe with right hand behind the head, and vice versa (scores can range from 2 to 6). The Fine Motor Movement factor included eight measures of motor functioning: pour water from a jug into a glass, pour water from one hand to the other (both dominant and non-dominant hands), insert key into lock and turn, insert electrical plug into socket, screw in a light bulb, put coins in a coin slot, and dial the numbers 1-9 on a rotary phone (scores can range from 8 to 24). The balance factor included ten measures of motor functioning and can also be considered a measure of gross motor function: walk and turn $3 \mathrm{~m}$, single chair stand, five chair stands, standing balance with feet side-by-side for up to $10 \mathrm{~s}$, standing balance with feet together and arms extended for up to $10 \mathrm{~s}$, lift a glass, lift a $1 \mathrm{~kg}$ packet, pick up a pen from the floor from a standing position, touch right fingers to left toes while seated, and vice versa (scores can range from 10 to 30). Mean scores on the motor factors at each wave are presented in Table 1.

For use in the statistical model, factor scores were transformed to T-score metric using mean and standard deviation at wave 1 to ensure a consistent metric; higher scores indicate more difficulties completing the tasks. Only two of the eight measures that make up the Fine Motor factor were collected at waves 8-10; therefore, fine motor factor scores could not be calculated for those waves of participation. The age range available for fine motor does not differ from the other motor factors; however, the coverage of data in the later ages is thinner. As a result, only 12 age intervals were used in modeling longitudinal trajectories for fine motor.

\section{Lung functioning}

Multiple measures of lung function are available; however, peak expiratory flow (PEF) taps intrinsic pulmonary function, whereas measures of vital capacity measure both lung function and respiratory muscle strength [20]. Lung function was tested on portable spirometers with subjects in seated position and their nasal passages blocked with nose clips. Two trials of PEF were completed and data from the best trial were used in the present analyses. At wave 2, forced expiratory volume in the first second (FEV1) was assessed instead of PEF. As both measures were available from all other waves, these data were used to create a linear transformation equation and FEV1 at wave 2 was translated to PEF units. Mean PEF values $(\mathrm{L} / \mathrm{min})$ at each wave are presented in Table 1. An apparent dip in PEF at IPT5 resulted from changes in the sample via both drop-out and the addition of new participants, as well as the longer interval between IPT3 and IPT5. As shown in Supplemental Table 1, when considered across age instead of wave, PEF demonstrates the expected monotonic decrease with age. For use in the statistical model, PEF was corrected for body volume through dividing it by the individual's squared height in meters [21] and the value was then transformed to T-score metric using mean and standard deviation at wave 1 . The purpose of the current analysis was to examine longitudinal covariation between lung and motor function, without over-correcting for possible contributions to mean-level function in either domain. Thus, we chose not to correct either lung or motor function for, say, smoking status, self-rated health, activity 
levels, etc. Correlations between PEF and the motor factors at each wave are presented in Supplemental Table 2. Across waves, lower scores on PEF were associated with more difficulties on all three motor factors.

\section{Statistical method}

Bivariate dual change score models (DCSM) were used to examine the bivariate relationship between lung and motor functioning. Extensive discussions of the model are available [22, 23], as well as comparisons of DCSM with latent growth curve models [24, 25]. As presented in Fig. 1, the model is based on latent difference scores that create a growth curve based on change from one age to another age $(\Delta y)$, which is modeled as a function of both constant change $(\alpha)$ that accumulates over time in an additive fashion and proportional change $(\beta)$ based on the previous score. Typically, $\alpha$ is set to 1 and the parameter $\beta$ differs from zero to the extent that the longitudinal change is nonlinear. The bivariate DCSM allows for a coupling mechanism $(\gamma$, see bolded paths in Fig. 1) where change in trait $X$ depends on the previous value of $Y$, and vice versa.

With the DCSM, it is possible to evaluate hypotheses about temporal order of changes in variables through restrictions on model parameters, while remaining agnostic as to underlying causes of the temporal relationship. Five alternative models were addressed. First, the relationship between the two variables may be bidirectional, such that $X$ affects changes in $Y$ and $Y$ affects changes in $X$ (i.e., both $\gamma_{\mathrm{yx}}$ and $\gamma_{\mathrm{yx}}$ are nonzero). Second, a model including no dynamic coupling among the variables was tested $\left(\gamma_{\mathrm{xy}}=\gamma_{\mathrm{yx}}=0\right)$. In subsequent models, the dynamic relationship in one direction only is tested $\left(\gamma_{\mathrm{yx}}=0\right.$ or $\left.\gamma_{\mathrm{xy}}=0\right)$ or the cross-variable dynamic effects are equated $\left(\gamma_{\mathrm{xy}}=\gamma_{\mathrm{yx}}\right)$.

Bivariate DCSM was fit to the data using Mplus Version 7.4 [26]. Model fit was indicated by the log-likelihood (-2LL) and the root-mean-square error of approximation (RMSEA). Adequate fit of the full model to the data is indicated when the RMSEA is less than or equal to 0.1 and an RMSEA of 0.05 or less indicates "close" fit [27]. Nested
Fig. 1 Bivariate dual change score model: $Y_{0}$ represents the intercept of the trajectory and $Y_{\mathrm{s}}$ represents the linear slope; $Y_{0} *$ and $Y_{\mathrm{s}}^{*}$ represent standardized intercept and slope. Mean $(\mu)$ and standard deviation $(\sigma)$ are estimated for each intercept and slope. $Y 50$ represents observed performance on measure $Y$ at age 50, with $y 50$ indicating the latent true score and $u_{\mathrm{y}} 50$ signifying error. Three-year age segments from $Y 50$ through $Y 86$ were included in the model. Error variance $\left(\sigma_{\mathrm{u}}\right)$ is assumed to be constant at each age. Change in performance $(\Delta y 53)$ is a function of constant change $(\alpha)$, proportional change $(\beta)$, and a coupling mechanism $(\gamma$ and bolded paths) where change in trait $X$ depends on the previous value of $Y$, and vice versa

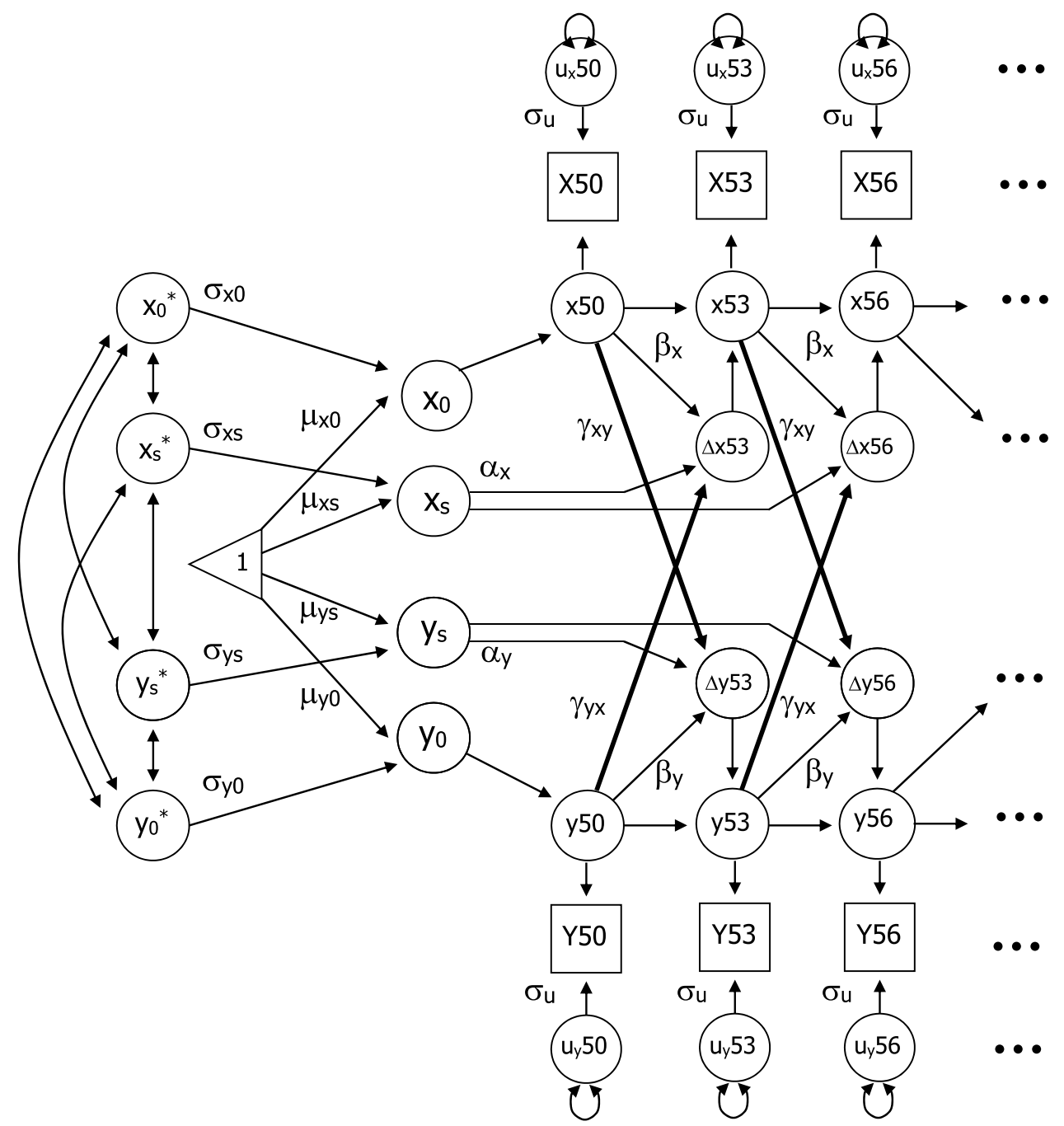


models were compared using the difference Chi-square test obtained by taking the difference between the obtained model fits (-2LL). The current analyses focused on individual performance by including a correction for twin pairs in the modeling.

\section{Results}

Model fit statistics for testing the five bivariate DCSM models are presented in Table 2. RMSEA indicated adequate fit of the model to the data for all three motor factors: for balance, RMSEA $=0.068$ (95\% CI $=0.065-0.071)$, for flexibility, RMSEA $=0.60$ (95\% CI $=0.057,0.063)$, and for fine motor, RMSEA $=0.060$ (95\% CI $=0.057-0.064)$. Comparing the no coupling model (model 2) to the full model (model 1) indicated significant reductions in model fit for the relationship between PEF and balance and between PEF and flexibility. No coupling was indicated for the relationship between PEF and fine motor. Additional models were tested to determine the nature of the bivariate relationship between lung function and the motor factors.

For the relationship of PEF with both Balance and Flexibility, setting the $\gamma$ motor $\rightarrow$ PEF parameter to zero (model 3) had no effect on model fit; whereas, setting the $\gamma \mathrm{PEF} \rightarrow$ motor parameter to zero (model 4 ) resulted in a

Table 2 Model fit statistics (-2LL) for the five comparison models testing the bivariate relationships between measures of motor function and PEF

\begin{tabular}{lllll}
\hline Models & Parameters & Balance & Flexibility & Fine motor \\
\hline 1: Full model & 21 & $-25,473$ & $-28,967$ & $-22,207$ \\
2: No coupling & 19 & $-25,485^{* *}$ & $-28,974^{*}$ & $-22,208$ \\
3: $\gamma$ Fac- & 20 & $-25,473$ & $-28,967$ & $-22,207$ \\
$\quad$ tor $\rightarrow$ PEF $=0$ & & & & \\
4: $\gamma$ PEF $\rightarrow$ fac- & 20 & $-25,482^{* *}$ & $-28,972^{*}$ & $-22,208$ \\
$\quad$ tor $=0$ & & & & \\
5: Equate cou- & 20 & $-25,481^{* *}$ & $-28,972^{*}$ & $-22,208$ \\
pling & & & & \\
\hline
\end{tabular}

* Change in model fit vs. the full model is significant at $p<0.05$

${ }^{* *}$ Change in model fit vs. the full model is significant at $p<0.01$ significant loss of fit compared with model 1 . Change in fit for model 4 versus model 1 was $9.02, d f=1, p<0.01$ for the balance factor and 4.71, $d f=1, p<0.05$ for the flexibility factor. For the Fine Motor factor, the change in fit for model 4 versus model 1 was not significant $(\Delta$ fit $=1.75$, $d f=1, n . s$.$) . Equating the coupling parameters provides an$ additional test of the direction of the relationship; beyond testing whether the parameters are significantly different from zero, it tests whether they are significantly different from each other. Again, this test (model 5 versus model 1) resulted significant changes in model fit for balance and flexibility, but not for the fine motor factor. Taken together, these results indicate that lung function precedes and contributes to subsequent changes in the balance and flexibility factors.

Parameter estimates and standard errors from the full bivariate model (model 1) are presented in Table 3. For each bivariate relationship, the estimate of the influence of PEF on the motor factor ( $\gamma \mathrm{PEF} \rightarrow$ factor) is larger than the estimate of the influence of the motor factor on PEF $(\gamma$ factor $\rightarrow$ PEF). Although it did not achieve significance for the fine motor factor, this difference between the coupling parameters was in the same direction as the other motor factors, such that $\gamma \mathrm{PEF} \rightarrow$ Fine Motor was larger than $\gamma$ fine motor $\rightarrow$ PEF. The longitudinal relationships between PEF and the motor factors are demonstrated in Fig. 1. Examining the difference in ageing trajectories for the full model versus the model without coupling parameters allows a visual representation of the impact of coupling. The decline trajectory for PEF is nearly identical both with and without coupling in all three bivariate relationships with the motor factors, illustrating the lack of impact the motor factors which have on subsequent age changes in lung function. In contrast, the differences between the ageing trajectories estimated by the full model and the no coupling model are evident for all three motor factors. In each case, when the impact of declining lung function is taken into account in the full coupling model, the increase in difficulties in motor function with age is reduced. The same pattern is evident even for the fine motor factor, although the lack of data at waves eight through ten resulted in truncated trajectories that did not achieve statistical significance. Thus, the bivariate DCSM indicates that lung function influences subsequent age

Table 3 Parameter estimates (SE) from the bivariate dual change score model between PEF and the motor factors (model 1)

\begin{tabular}{|c|c|c|c|c|c|c|}
\hline Parameters & $\begin{array}{l}\text { PEF (with Bal- } \\
\text { ance) }\end{array}$ & Balance & $\begin{array}{l}\text { PEF (with Flex- } \\
\text { ibility) }\end{array}$ & Flexibility & $\begin{array}{l}\text { PEF (with Fine } \\
\text { motor) }\end{array}$ & Fine motor \\
\hline Mean intercept, $\mu_{0}$ & $56.14(0.57)$ & $49.37(0.62)$ & $56.47(0.54)$ & $53.72(1.35)$ & $56.06(0.54)$ & $47.27(0.50)$ \\
\hline Mean slope, $\mu_{\mathrm{s}}$ & $-2.60(1.66)$ & $-1.50(4.61)$ & $-2.03(1.31)$ & $5.22(6.68)$ & $0.02(4.44)$ & $-35.42(8.33)$ \\
\hline Proportional change, $\beta$ & $0.03(0.02)$ & $0.36(0.03)$ & $0.02(0.02)$ & $0.26(0.03)$ & $0.03(0.04)$ & $0.58(0.09)$ \\
\hline Coupling, $\gamma \mathrm{PEF} \rightarrow$ factor & & $-0.30(0.07)$ & & $-0.35(0.11)$ & & $0.15(0.08)$ \\
\hline Coupling, $\gamma$ factor $\rightarrow$ PEF & $-0.00(0.01)$ & & $-0.00(0.01)$ & & $-0.05(0.06)$ & \\
\hline Error deviation, $\sigma_{\mathrm{u}}$ & $5.71(1.03)$ & $9.29(1.64)$ & $5.68(1.02)$ & $23.46(3.96)$ & $5.56(1.02)$ & 9.09 (1.74) \\
\hline
\end{tabular}


changes in motor function, such that reduced lung capacity contributes to more difficulties in motor function at subsequent testing waves.

\section{Discussion}

To systematically test the chain of events predicted by the disablement process, dual change score models were used to test the temporal relationships between lung function and motor function in longitudinal data. Comparison of models incorporating influences from lung function to subsequent motor function and from motor function to subsequent lung function indicated clearly that the chain of events moved in one direction: reduction in lung function contributes to subsequent increases in difficulties in motor function. Possible mechanisms for this relationship include systemic changes that affect both lung function and motor function [16] or more specific influences of declining lung function on the vigor required to complete motor function tasks $[7,8]$.

It is important to consider the connection between lung function and motor function as one link in a larger chain of events. A wider-angle interdisciplinary lens, incorporating both physical and cognitive components, is required to fully understand the trajectory of functional consequences that over time lead from independence to dependence [6, 28]. Studies have found relationships among personality, cognition, lung function, and motor function in various configurations of predictor and outcome in both cross-sectional and longitudinal analyses $[3,10,12]$. Piecing together, the individual links in the chain from multiple perspectives can provide a possible model for the cascade of events that tend to result in disablement and dependence. For example, a recent meta-analysis examining the results of 40 longitudinal studies of the relationship between physical and cognitive functioning found stronger evidence that baseline physical function predicted changes in cognitive function than the reverse [29]. Similarly, a coordinated analysis of eight longitudinal studies of lung function and cognitive function found a consistent link between changes in the two domains [30]. In addition, the previous applications of the dual change score model to SATSA data have indicated separate unidirectional steps in a possible chain events: processing speed contributed to subsequent changes in cognitive function [31], lung function contributed to subsequent changes in processing speed [32], and motor function contributed to subsequent changes in processing speed [33]. The current analysis adds to a growing body of literature indicating that lung function contributes to subsequent motor function and mobility. Taken together, these studies suggest a possible cascade of events: declining lung function results in increasing difficulties in motor function, which contribute to subsequent declines in processing speed, which underlie changes associated with cognitive ageing. The end result, arising from both physical and cognitive changes, will be reduced independence and greater reliance on assistance in some form.

Although DCSM is considered to have many important advantages over other methods for addressing hypotheses about dynamic relationships among variables [34], the method is also limited by many of the statistical assumptions common to structural equation models. The data are assumed to be missing at random, the sample is assumed to be relatively homogeneous, and structural relations based on interindividual variance and on intraindividual variance are assumed to be equivalent [25]. Differences between the two major variables in reliability, change with age, or measurement level could impact the fit of the bivariate DCSM. Particularly relevant here is the difference in measurement level between lung function and motor function. PEF measures an individual's maximal effort, which may be more sensitive to changes with age. The motor factor measures that had a maximal time limit (e.g., balance for $10 \mathrm{~s}$ ) by definition did not measure an individual's maximal effort. Only 2 of the 20 measures of motor functioning would be subject to this limitation; however, nurse ratings of performance will not have the same level of rigor and reliability that a physiological measure like PEF has. Nurse ratings were used instead of time to complete the tasks, because they demonstrated more sensitivity to performance difficulties across the range of ages included in SATSA than the timing measures. Moreover, as demonstrated in Fig. 2, the motor factors demonstrated increases in difficulty with age greater in magnitude than the age changes shown by PEF. As a result, conclusions from the bivariate DCSM should be interpreted with some caution.

As with any longitudinal sample, attrition occurred in SATSA. Even though the sample was representative of the population at intake, non-random drop-out through the course of the longitudinal studies results in increasingly select samples of adults who are healthy enough to participate. In SATSA, research nurses visited the participants at their current residence; therefore, data collection could continue even after onset of illness or entry in to care. As a result, wave-to-wave drop-out was quite low (about $8 \%$ ), but drop-out accumulates across waves. Consequently, our analyses have likely underestimated the extent of change with age.

The use of qualitative assessment of motor functioning instead of timed assessment could be considered both a strength and a weakness of the current analyses. Qualitative assessment of performance by trained nurses produced more nuanced assessment of physical performance: for example, a stopwatch does not capture the "wobbles" that an observer can see during a balance task. Similarly, reports of fatigue or increased time required to complete activities of daily 

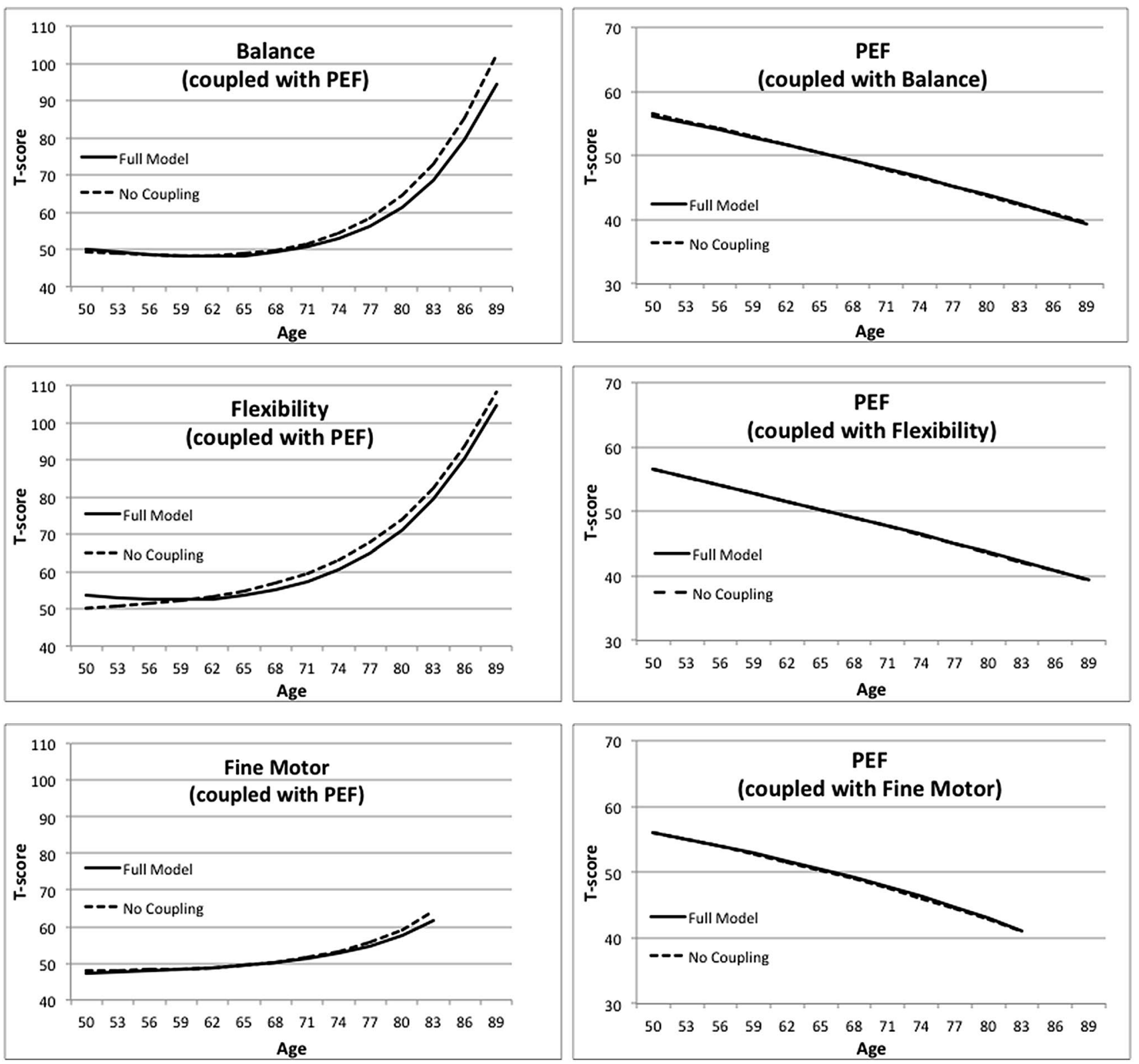

Fig. 2 Change trajectories estimated for the motor factors and PEF by the model, with and without coupling

living can be more informative than simple success or failure [8]. The qualitative assessment used here resulted in sufficient variability to support factor analysis [18] and structural modeling across the entire age range included in the current analyses. However, regardless of how well trained the nurseinterviewers were and how little staff turnover occurred during SATSA (12 interviewers over 27 years), observer ratings are unlikely to be as reliable as timed measures of performance.

Combined with previous results, these results suggest a pathway that may start with age declines in lung function, impact motor function, and then processing speed, finally resulting in cognitive ageing. These results indicate that assessments of older persons, such as the Comprehensive Geriatric Assessments [35], should include lung function to identify individuals at risk at an earlier stage. Moreover, if reduced pulmonary function is identified, it should lead to a more detailed evaluation of motor function and a search for factors that may influence both lung and motor function. In turn, interventions focusing on improving or maintain lung function should have the added effect of maintaining motor and cognitive function and delaying the onset of dependence and need for care. Whereas in previous centuries, only the most vigorous (and luckiest) individuals survived to late old age, health advances of the twentieth century have allowed frailer and more vulnerable individuals to live longer $[2,36]$. 
Appropriate treatment and strategic interventions can ensure that longer life does not necessarily mean a longer period of morbidity and decline $[1,6]$. Our results suggest that interventions focused on lung function could have positive impacts on functioning in multiple domains. Understanding the cascade of events that can lead to dependence can help in the development of interventions targeted early in the disablement process.

Acknowledgements Open access funding provided by Jönköping University. Swedish Adoption/Twin Study of ageing was supported by Grants National Institutes of Health AG04563, R01 AG10175, the MacArthur Foundation Research Network on Successful ageing, the Swedish Council For Working Life and Social Research (FAS) (97:0147:1B, 2009-0795), and Swedish Research Council (825-20077460, 825-2009-6141).

Author contributions All authors contributed to the study conception and design. Data analyses were performed by DF. The first draft of the manuscript was written by DF and MEB and all authors commented on previous versions of the manuscript. All authors read and approved the final manuscript.

\section{Compliance with ethical standards}

Conflict of interest The authors declare that they have no conflicts of interest.

Ethical approval All procedures have been reviewed by a physician affiliated with the study and all have been approved by the Ethics Committee at Karolinska Institutet (dnr. 84:61, dnr 98:319) and the Regional Ethics Review Board (dnr. 2010/657-31/3). All procedures were in accordance with the 1964 Helsinki declaration and its later amendments or comparable ethical standards.

Informed consent Informed consent was obtained from all individual participants in the study.

Open Access This article is licensed under a Creative Commons Attribution 4.0 International License, which permits use, sharing, adaptation, distribution and reproduction in any medium or format, as long as you give appropriate credit to the original author(s) and the source, provide a link to the Creative Commons licence, and indicate if changes were made. The images or other third party material in this article are included in the article's Creative Commons licence, unless indicated otherwise in a credit line to the material. If material is not included in the article's Creative Commons licence and your intended use is not permitted by statutory regulation or exceeds the permitted use, you will need to obtain permission directly from the copyright holder. To view a copy of this licence, visit http://creativecommons.org/licenses/by/4.0/.

\section{References}

1. Fulop T, Larbi A (2018) Biology of aging: paving the way for healthy aging. Exp Gerontol 107:103

2. Hoogendijk EO, van der Noordt M, Onwuteaka-Philipsen BD et al (2019) Sex differences in healthy life expectancy among nonagenarians: a multistate survival model using data from the vitality 90+ study. Exp Gerontol 116:80-85
3. Singh-Manoux A, Dugravot A, Kauffman F et al (2011) Association of lung function with physical, mental and cognitive function in early old age. Age 33:385-392. https://doi.org/10.1007/s1135 7-010-9189-x

4. Sloane PD, Marzetti E, Landi F et al (2019) Understanding and addressing muscle strength, mass, and function in older persons. JAMDA 20:1-4

5. den Ouden MEM, Schuurmans MJ, Arts IE et al (2011) Physical performance characteristics related to disability in older persons: a systematic review. Maturitis. 69:208-219. https://doi. org/10.1016/j.maturitas.2011.04.008

6. Verbrugge LM, Jette AM (1994) The disablement process. Soc Sci Med 38:1-14

7. Buchman AS, Boyle PA, Leurgans SE et al (2009) Pulmonary function, muscle strength, and incident mobility disability in elders. Proc Am Thorac Soc 6:581-587. https://doi.org/10.1513/ pats.200905-030RM

8. Schroll M (1994) The main pathway to musculoskeletal disability. Scand J Med Sci Sports 4:3-12

9. Butcher SJ, Meshke JM, Sheppard MS (2004) Reductions in functional balance, coordination, and mobility measures among patientis with stable chronic obstructure pulmonary disease. J Cardpulm Rehabil 24:274-280

10. Cook NR, Albert MS, Berkman LF et al (1995) Interrelationships of peak expiratory flow rate with physical and cognitive function in the elderly: MacArthur Foundation Studies of Aging. J Gerontol Med Sci 50A:M317-M323

11. Watsford ML, Murphy AJ, Pine MJ (2007) The effects of ageing on respiratory muscle function and performance in older adults. J Sci Med Sport 10:36-44

12. Femia EE, Zarit SH, Johansson B (1997) Predicting change in activities of daily living: a longitudinal study of the oldest old in Sweden. J Gerontol Psychol Sci 52B:P294-P302

13. Schroll M, Avlund K, Davidsen M (1997) Predictors of 5-year functional ability in a longitudinal survey of men and women aged 75-80: The 1914-population in Glostrup, Denmark. Aging Clin Exp Res 9:143-152

14. Buchman AS, Boyle PA, Leurgans SE et al (2008) Respiratory muscle strength predicts decline in mobility in older persons. Neuroepidemiology 31:174-180. https://doi.org/10.1159/00015 4930

15. Warburton DER, Nicol CW, Bredin SSD (2006) Health benefits of physical activity: the evidence. CMAJ 174:801-809. https:// doi.org/10.1503/cmaj.051351

16. Sillanpää E, Stenroth L, Bijlsma AY et al (2014) Associations between muscle strength, spirometric pulmonary function and mobility in healthy older adults. Age (Dordr) 36:9667. https:// doi.org/10.1007/s11357-014-9667-7

17. Finkel D, Pedersen NL (2004) Processing speed and longitudinal trajectories of change for cognitive abilities: The Swedish Adoption/Twin Study of Aging. Ageing Neuropsychol Cognit 11:325-345. https://doi.org/10.1080/13825580490511152

18. Ernsth Bravell M, Finkel D, Dahl Aslan A et al (2017) Motor functioning differentially predicts mortality in men and women. Arch Gerontol Geriatr 72:6-11. https://doi.org/10.1016/j.archg er.2017.05.001

19. Ernsth-Bravell M, Zarit S, Johansson B (2011) Self reported activities of daily living and performance based functional ability: a study of congruence among the oldest old. Eur J Ageing 8:199-209. https://doi.org/10.1007/s10433-011-0192-6

20. Buchman AS, Boyle PA, Wilson RS et al (2008) Pulmonary function, muscle strength and mortality in old age. Mech Ageing Dev 129:625-631. https://doi.org/10.1016/j.mad.2008.07.003

21. Sternäng O, Palmer K, Kabir ZN et al (2018) Associations between functional biological age and cognition among older 
adults in rural Bangladesh: comparisons with chronological age. J Ageing Health. https://doi.org/10.1177/0898264318757147

22. McArdle JJ (2001) A latent difference score approach to longitudinal dynamic structural analyses. In: Cudeck R, Dutoit S, Sorbom $\mathrm{S}$ (eds) Structural equation modeling: present and future. Scientific Software International, Lincolnwood, pp 342-380

23. McArdle JJ, Hamagami F, Meredith W et al (2000) Modeling dynamic hypothesses of Gf-Gc theory using longitudinal lifespan data. Learn Individ Differ 12:53-79

24. Ghisletta P, de Ribaupierre A (2005) A dynamic investigation of cognitive dedifferentiation with control for retest: evidence from the Swiss Interdisciplinary Longitudinal Study of the Oldest Old. Psychol Ageing 20:671-682

25. Lövdén M, Ghisletta P, Lindenberger U (2005) Social participation attenuates decline in perceptual speed in old and very old age. Psychol Ageing 20:423-434

26. Muthén LK, Muthén BO (2012) Mplus user's guide, 7th edn. Muthén \& Muthén, Los Angeles

27. Browne M, Cudeck R (1993) Alternative ways of assessing model fit. In: Bollen K, Long S (eds) Testing structural equation models. Sage, Beverly Hills, pp 136-162

28. Alwin DF, Hofer SM (2008) Opportunities and challenges for interdisciplinary research. In: Hofer SM, Alwin DF (eds) Handbook of cognitive aging: interdisciplinary perspectives. SAGE, Los Angeles, pp 2-31

29. Clouston SAP, Brewster P, Kuh D et al (2013) The dynamic relationship between physical function and cognition in longitudinal aging cohorts. Epidemiol Rev 35:33-50
30. Duggan EC, Piccinin AM, Clouston S et al (2019) A multi-study coordinated meta-analysis of pulmonary function and cognition in aging. J Gerontol Med Sci 74:1793-1804. https://doi.org/10.1093/ gerona/glz057

31. Finkel D, Reynolds CA, McArdle JJ et al (2007) Age changes in processing speed as a leading indicator of cognitive aging. Psychol Ageing 22:558-568

32. Emery CF, Finkel D, Pedersen NL (2012) Pulmonary function as a leading cause of cognitive aging. Psychol Sci 23:1024-1032

33. Finkel D, Ernsth-Bravell M, Pedersen NL (2016) Temporal dynamics of motor functioning and cognitive aging. J Gerontol Med Sci 71:109-116

34. Ghisletta P, Lindenberger U (2003) Age-based structural dynamics between perceptual speed and knowledge in the Berlin Aging Study: direct evidence for ability dedifferentiation in old age. Psychol Ageing 18:696-713

35. Tuttle CSL, Maier AB (2018) Towards a biological geriatric assessment. Exp Gerontol 107:102-107

36. Vaupel JW, Yashin AI (1985) Heterogeneity's ruses: some surprising effects of selection on population dynamics. Am Stat 39:176-185

Publisher's Note Springer Nature remains neutral with regard to jurisdictional claims in published maps and institutional affiliations. 